\title{
Acceso a la educación superior: Uruguay y Ecuador Estudio de derecho comparado
}

\author{
Access to higher education: Uruguay and Ecuador \\ Comparative Law study
}

\author{
Rina Pazos \\ Universidad Tecnológica Equinoccial (UTE) \\ Universidade do Minho \\ rinapazos986@hotmail.com \\ Código Orcid: https://orcid.org/0000-0002-2522-8138
}

\begin{abstract}
Resumen
En el mundo contemporáneo la educación superior se ha tornado un elemento de gran importancia no solo desde la perspectiva de los derechos individuales, sino también como parte de la estrategia de desarrollo de los Estados. El Pacto Internacional de Derechos Económicos, Sociales y Culturales (PIDESC, 1966) reconoce a la educación como un derecho de las personas y establece que el acceso igualitario y la implantación progresiva de la gratuidad en la educación superior son necesarios para lograr el pleno ejercicio de ese derecho. En ese marco, los ordenamientos jurídicos de la materia dan importantes insumos para conocer los modelos sobre acceso a la educación superior de cada país. El presente trabajo tiene por objetivo aproximarse a los modelos de dos países de América Latina: Uruguay y Ecuador, a través de sus ordenamientos jurídicos en la materia; para ello se ha adoptado el "método del derecho comparado" que permite realizar un análisis de las diferentes fuentes del Derecho de los Estados a comparar, para alcanzar una síntesis que brinde evidencia de las semejanzas y diferencias sobre el contenido jurídico, valores y modelos políticos sobre los que se asientan. Ambos Estados, a través de sus normas, consideran a la educación superior como un bien público y un derecho, las diferencias se reflejan en los mecanismos de implementación del acceso a ese derecho.
\end{abstract}

\section{Palabras clave}

Derecho comparado, derecho a la educación, democratización de la educación, enseñanza superior, enseñanza gratuita, oportunidades educacionales.

Forma sugerida de citar: Pazos, Rina (2018). Acceso a la educación superior: Uruguay y Ecuador, estudio de derecho comparado. Universitas, 29, pp. 157-176. 


\begin{abstract}
Nowadays, higher education has become an important element not only from the individual rights perspective but also as part of the development strategy of many countries. The International Covenant on Economic, Social and Cultural Rights (ICESCR, 1966) recognizes education as an individual's right and establishes that equal access and progressive free education in higher education is necessary to achieve the full exercise of that right. In this framework, the legal norms in the field give important inputs to understand the access patterns to higher education in each country. The aim of this paper is to approach to the models in two Latin American countries: Uruguay and Ecuador, through the scrutiny of their regulations on this matter; the Comparative Law method has been adopted, which allows an analysis of the different sources of Law to be compared to achieve a synthesis that provides evidence of the similarities and differences on their legal content, values and political models. Both legal systems consider higher education as a public good and a right, the differences are reflected in the mechanisms for implementing access to that right.
\end{abstract}

\title{
Keywords
}

Comparative Law, right to education, democratization of education, higher education, free education, educational opportunities.

\section{Introducción}

En el mundo contemporáneo la formación del talento humano - también llamado capital humano - tiene relevancia no solamente en el plano del derecho individual de las personas, sino también como una estrategia de desarrollo de los Estados, tanto desde las perspectivas liberales como desde las socialistas/comunistas.

El Pacto Internacional de Derechos Económicos, Sociales y Culturales (PIDESC, 1966) reconoce a la educación como un derecho de las personas y establece que el acceso igualitario y la implantación progresiva de la gratuidad en la educación superior, son necesarias para lograr el pleno ejercicio de ese derecho. Para los países de América Latina, la educación superior implica un reto y un tema ineludible en el mundo actual. Uno de los desafíos de la región es la masificación con equidad para la promoción y movilidad social (UNESCO, 2015, p. 90). 
Los ordenamientos jurídicos seleccionados para realizar la comparación son el ecuatoriano y el uruguayo. Esta elección se realizó considerando:

- Que los ordenamientos jurídicos de ambos países están insertados en la misma tradición jurídica, es decir, en la tradición civilística, lo cual hace más viable la comparación de las instituciones al estar asentadas sobre los mismos fundamentos jurídicos.

- Ambos países reconocen y garantizan la gratuidad de la educación superior pública, una semejanza inusual sobre la cual resulta interesante indagar con mayor profundidad.

- Ambos países se encuentran en la misma región geográfica, lo cual puede sugerir que existen factores históricos y sociales coincidentes.

- El tamaño de los sistemas de educación superior a comparar es similar en relación al de otros países de la región que mantienen las características detalladas en los numerales anteriores.

Si bien existen otros países en América Latina que mantienen sistemas públicos de educación superior gratuitos, como el caso de Argentina y Brasil (UNESCO, 2018), sus dimensiones son mucho mayores que los sistemas de educación superior ecuatoriano y uruguayo (UNESCO, 2018), una variable que exigiría otro tipo de abordaje para sus problemas propios de su tamaño y, más aún, si se considera sus modelos federales de gobierno.

El método que se utiliza en esta investigación es el descrito por De Almeida Ferreira y Carvalho Morais (2017, pp. 26-36), en el que se sugiere realizar la micromparación a través de la idea gráfica de una parrilla comparativa con un eje sintagmático (en el que se incluyen las dimensiones histórica, metajurídica y jurídica del tema a comparar) y un eje paradigmático (en el que se desarrollarán las variaciones de las dimensiones sintagmáticas). El proceso incluye una fase analítica y una integrativa, para finalizar con una síntesis comparativa. Siguiendo lo propuesto por Moura Vicente (2012, p. 39), se intenta identificar las semejanzas y diferencias entre los dos sistemas $\mathrm{y}$ encontrar algunas causas.

Como ya se anticipó, el presente trabajo plantea el abordaje del objeto de estudio a través del "método del derecho comparado", que implica la comparación sistemática de derechos u ordenamientos jurídicos para identificar semejanzas y diferencias (De Almeida y Carvalho, 2017, p. 11). Específicamente, se realizará un ejercicio microcomparativo que consiste en la 
comparación de temas o instituciones jurídicas afines en ordenamientos jurídicos distintos, considerando a una institución jurídica como el conjunto de normas, principios y organizaciones de naturaleza jurídica que forman parte de un determinado ordenamiento jurídico y constituyen una unidad basada en aspectos sociales, jurídicos o doctrinarios (De Almeida y Carvalho, 2017, p. 13). La aproximación de la microcomparación será funcional, es decir, se selecciona y compara una institución jurídica que en diferentes sistemas jurídicos da soluciones jurídicas a necesidades similares (De Almeida y Carvalho, 2017, p. 27).

El objeto de la comparación es el acceso a la educación superior desde su dimensión jurídica, en dos ordenamientos jurídicos distintos con un criterio temporal actual y sincrónico, pero incorporando una perspectiva histórica y considerando los aspectos que sean susceptibles de ser comparados.

\section{Dimensión histórica}

\section{Evolución histórica de la educación superior en Uruguay}

La fundación de la universidad en Uruguay inicia con la creación de la Casa de Estudios Generales en 1833, transformada en Universidad de la República de Uruguay en 1838. Los siguientes treinta años la universidad se caracterizó, según Contera (2008, p. 535), por la corriente filosófica del espiritualismo ecléctico que sirvió para mantener una línea anticlerical y liberal.

En el último tercio del siglo XIX inició el periodo positivista con la creación de la Facultad de Medicina y Matemáticas, modelo que fue impulsado por José Pedro Varela, cuyo proyecto se caracterizaba por lograr que la educación fuese obligatoria, gratuita y laica (Consejo de Educación Primaria, 2018). En la primera mitad del siglo XX se avanzó hacia una universidad profesionalista y en la Constitución de 1934 se declaró de utilidad social la gratuidad de la enseñanza oficial (art. 62); además, en la Constitución de 1952 se estableció la forma de designación del Consejo Directivo de la Universidad de la República en la que participarían estudiantes, docentes y egresados (art. 205) y se declaró la autonomía de las instituciones de enseñanza pública (art. 204). La segunda mitad del siglo XX inició con la consagración de la autonomía académica y política en Ley Orgánica de la Universidad de la República (1958), impulsada por las movilizaciones 
estudiantiles influenciadas por el Movimiento Autonomista de Córdoba en Argentina (1918). En la Constitución de 1967 se garantizó la libertad de enseñanza. En 1973, la dictadura militar silenció a la institución y destituyó a varios profesores; en ese periodo también empezó un proceso de masificación de la matrícula, pasando de 16 mil alumnos en los años 70 a 63 mil en 1988. En 1980, terminada la dictadura, la Universidad de la República de Uruguay pudo volver a repensarse y a decidir por sí misma, a través de la recuperación del sentido y hábito de la crítica (Martínez, 2003, pp. 3-12).

Sobre la historia más reciente, Contera $(2008$, p. 535) señala que el proceso de privatización de la educación superior en Uruguay inició en 1984, con la creación de la Universidad Católica de Uruguay, y continuó en la década de los 90 con algunas más, sin embargo, la mayor parte de la matrícula universitaria se concentra en la Universidad de la República.

\section{La evolución histórica de la educación superior en el Ecuador}

La educación superior en el Ecuador tiene sus orígenes en la Real Audiencia de Quito, durante la Colonia española. Desde 1620 funcionaban en el territorio de lo que hoy es el Ecuador tres universidades y en 1779 todas las universidades de Quito se unificaron con el nombre de Universidad de Santo Tomás, por iniciativa del obispo José Domingo Pérez Calama (Pacheco, 2015, pp. 175-178). Luego de la Independencia, el modelo napoleónico profesionalizante es el que se importó para la educación superior.

A esta etapa le siguieron tres reformas importantes. La primera reforma fue la del liberalismo y la secularidad, que inició en 1907, cuando el Estado adoptó un rol de financiamiento y se avanzó hacia la inclusión de los mestizos (Guijarro, 2016, pp. 220-221). La segunda, fue de reflexividad y crítica, desde mediados del siglo XX, en la que cobró una mayor relevancia la dimensión social de la universidad, marcada por la organización estudiantil influida por el Movimiento Autonomista de Córdoba en Argentina (1918), la Revolución Cubana (1958), la Revolución Cultural China (1966) y los eventos de Mayo del 68 en Francia. Este periodo estuvo marcado por la búsqueda de una mayor democratización del acceso a las aulas universitarias, una mayor autonomía institucional frente al Estado y una verdadera función social (Guijarro, 2016, pp. 224-226). Finalmente, La tercera reforma se gestó a fines de la primera década del siglo XXI, en el escenario de la globalización 
y el neoliberalismo, cuyos efectos más notorios se observaron entre 1980 y mediados de la primera década del siglo XXI, periodo durante el cual la universidad sufrió empobrecimiento y privatización (Ramírez, 2013, p. 17). De tal forma que entre 1998 y 2000 se crearon quince universidades, de las cuales trece eran particulares autofinanciadas y dos públicas (Minteguiaga y Ramírez, 2010,p. 138). Esta reforma llegó de la mano con la ola de Gobiernos progresistas en América Latina, que en Ecuador inició en 2007, lo cual implicó un nuevo proceso constituyente y una agenda política y programática de recuperación del Estado, la inversión pública y los derechos, bajo el paradigma del Buen Vivir. En ese marco, la actual Constitución expedida en 2008 declaró la gratuidad de la educación superior hasta el tercer nivel y el establecimiento de un sistema de nivelación y admisión para el sistema público de educación superior.

\section{Dimensión metajurídica}

\section{Características socioeconómicas de Uruguay}

Uruguay es una república democrática con una extensión territorial continental de $176215 \mathrm{~km}^{2}$ y está ubicada en la costa este de América Latina. Cuenta con una población de 3440157 habitantes (INE, 2014, pp. 17, 22), donde más del $90 \%$ de su población se considera blanca (INE, 2018). En general, es considerada como la sociedad con mayores avances en temas de igualdad en América Latina: la brecha entre ricos y pobres visibilizada a través del coeficiente de Gini por ingreso ${ }^{1}$ ha bajado desde 0,47 en 2006 a 0,41 en 2015 (BM, 2018); es decir, hay una importante reducción de la desigualdad en ese periodo, aunque no alcanza el nivel de los países de la OCDE, cuyo promedio en 2014 fue 0,318 (OCDE, 2018). Sin embargo, tiene el ingreso per cápita más alto de América Latina y una clase media del $60 \%$ de su población (BM, 2018). Su PIB pasó de 19,5 mil millones a 52,4 mil millones de dólares entre 2006 y 2016 (BM, 2018) y sus exportaciones se con-

1 Según el Sistema Integrado de Indicadores Sociales del Ecuador, el coeficiente de Gini del ingreso es una medida estadística de la desigualdad en la distribución del ingreso de los perceptores individuales, que varía entre 0 y 1 . Muestra mayor desigualdad mientras se aproxima a 1 y corresponde a 0 en el caso hipotético de una distribución totalmente equitativa. 
centran en productos como carne, cereales, leche y lácteos, madera y manufacturas (Instituto Uruguay XXI, 2011).

La inversión pública de Uruguay en educación superior fue del 1,2\% sobre el PIB en el año 2011 (Ballas, 2016,p. 90) y su tasa bruta de matrícula en la educación superior ${ }^{2}$ tuvo un importante crecimiento entre 2006 y 2010 , pasando de 46,03 a 63,13 (BM, 2018).

\section{Características socioeconómicas de Ecuador}

En cuanto a los indicadores sociales, el Ecuador cuenta con un territorio de $256370 \mathrm{~km}^{2}$ y tiene una población de 14483499 habitantes según el censo poblacional de 2010 (INEC, 2018). El 21,6\% se considera montubio, afroecuatoriano o indígena, el 71,9\% mestizo y el 6,1\% blanco. En general, es una sociedad desigual, sin embargo, han existido esfuerzos para reducir la brecha entre ricos y pobres. Así, el coeficiente de Gini por ingreso pasó de 0,54 en 2006 a 0,47 en 2015 (SIISE, 2018), es decir, hubo una importante reducción de la desigualdad en ese periodo, aunque no se alcanzó el nivel de los países de la OCDE, cuyo promedio en 2014 fue 0,318 (OCDE, 2018).

La economía del país es eminentemente primario-exportadora y secundario-importadora. Su principal recurso es el petróleo con lo cual depende en gran medida de su precio en el mercado internacional. A pesar de los esfuerzos realizados durante los últimos años, en que el PIB pasó de 51 millones de dólares a 98 millones de dólares 3 (BCE, 2018), las exportaciones no petroleras solo crecieron un $14 \%$ entre 2012 y 2016, y esto debido a la caída de los precios del petróleo en los últimos años (BCE, 2018); sin embargo, en la época de la bonanza por los altos precios del petróleo, tales ingresos fueron invertidos en la reducción de la pobreza (Ramírez, 2017, p. 84) para que la crisis no afectara a los estratos más pobres (Ramírez, 2017).

En lo que respecta al acceso a la educación superior, la matrícula creció en 136 mil estudiantes, entre 2006 y 2014, lo que implicó el incremento de 4,1 puntos porcentuales del total y corresponde al doble del crecimiento

2 Tasa bruta de matrícula, educación superior (niveles 5 y 6 de la CINE), total. Corresponde al número total de estudiantes matriculados en educación superior (niveles 5 y 6 de la CINE), independientemente de su edad, expresado como porcentaje de la población total del grupo etario cinco años después de finalizar la enseñanza secundaria (BM, 2018).

3 Años comparados: 2007 y 2016. 
anual poblacional. La probabilidad de ingresar a la universidad siendo pobre en 2006 era del 33\% y en 2014 esa probabilidad aumentó al 67\% (Ramírez, 2016, p. 26).

\section{Dimensión jurídica}

\section{Fuentes del derecho sobre la educación superior}

Las fuentes de derecho que tratan sobre los aspectos de la educación terciaria en Uruguay son las siguientes normas en vigor (enumeradas jerárquicamente): la Constitución de la República del Uruguay (CRU), el PIDESC, la Ley General de Educación (LGE), las leyes de creación de las universidades públicas (Universidad de la República de Uruguay y la Universidad Tecnológica de Uruguay), la ley de presupuesto para el periodo 2016-2021, el Decreto Ley $\mathrm{N}^{\mathrm{o}} 15661$ del 20 de noviembre de 1984 referido a las universidades privadas y el Decreto Ley No 104/014 aprobado el 28 de abril de 2014 que trata sobre las autorizaciones de funcionamiento y registro de carreras de las instituciones de educación terciaria universitaria y no universitaria privadas.

La costumbre no es fuente del derecho en el sistema jurídico uruguayo excepto en los casos que la ley se remite a ella (González et al., 2017, p. 25). No se ha identificado la existencia de litigios que hayan dado lugar a sentencias relacionadas con el ámbito de la enseñanza terciaria. La jurisprudencia no constituye fuente de derecho en el sistema jurídico uruguayo conforme el artículo 12 de su Código Civil, el cual señala: "Solo toca al legislador explicar o interpretar la ley, de un modo generalmente obligatorio. Las sentencias judiciales no tienen fuerza obligatoria sino respecto de las causas en que actualmente se pronunciaren" (en González et al., 2017, p. 26).

En el caso de Ecuador, las fuentes normativas de la educación superior son: la Constitución de la República del Ecuador (CRE), el PIDESC, la Ley Orgánica de Educación Superior (LOES), el Reglamento General a la Ley Orgánica de Educación Superior (RGLOES), la Ley del Fondo de Desarrollo Universitario y Politécnico (FOPEDEUPO), los reglamentos expedidos por el Consejo de Educación Superior (CES) y los reglamentos expedidos por el Consejo de Evaluación, Acreditación y Aseguramiento de la Calidad de la Educación Superior (CEAACES). 
La costumbre no es fuente del derecho en el sistema jurídico ecuatoriano excepto en los casos que la ley se remite a ella tal como lo establece el Código Civil ecuatoriano en su artículo 2. La jurisprudencia constituye fuente de derecho en el sistema jurídico ecuatoriano cuando proviene de parámetros interpretativos de la CRE fijados por la Corte Constitucional. El artículo 2 de la Ley de Garantías Jurisdiccionales y Control Constitucional señala la obligatoriedad del precedente constitucional de interpretación de la CRE; sin embargo, no se ha identificado la existencia de jurisprudencia relacionada con la educación superior.

\section{Categoría de la educación superior en el ordenamiento jurídico de Uruguay y Ecuador}

\section{Uruguay: bien público y derecho fundamental en la ley}

En Uruguay, la educación en general, incluyendo la educación superior - denominada como educación terciaria - es considerada por la LGE como un bien público y un derecho fundamental (arts. 1 y 2 ). Además, se comprende como "el conjunto de propuestas educativas integradas y articuladas para todos los habitantes a lo largo de la vida" (art. 20). Dentro de este sistema se sitúa la educación terciaria como un nivel de formación (art. 22). Sus principios son: universalidad (art. 6), obligatoriedad (art. 7), diversidad e inclusión educativa (art. 8), participación (art. 9), libertad de enseñanza (art. 10), libertad de cátedra (art. 11), autonomía (art. 46), coordinación (art. 47). Por su lado, la CRU garantiza la libertad de enseñanza (art. 68) y declara de utilidad social la gratuidad de la enseñanza superior (art. 71).

\section{Ecuador: bien público y derecho constitucional}

En el Ecuador la educación superior es considerada como un bien público, además de un derecho. La CRE establece que "la educación es un derecho de las personas a lo largo de su vida y un deber ineludible e inexcusable del Estado" (art. 26); además, determina claramente que esta constituye la garantía de la igualdad e inclusión social. El artículo 28 de la CRE señala que "la educación pública será universal y laica en todos sus niveles, y gratuita hasta el tercer nivel de educación superior". A nivel legal, el artículo 2 de la LOES determina que la educación superior es un derecho de 
las personas y un bien público y social. Nuevamente, la misma CRE señala la existencia del sistema de educación superior, conformado por las universidades, escuelas politécnicas, institutos superiores técnicos, tecnológicos y pedagógicos, y conservatorios de música y artes (art. 350). Los principios por los que se rige el sistema que son: autonomía responsable, cogobierno, igualdad de oportunidades, calidad, pertinencia, integralidad, autodeterminación para la producción del pensamiento y conocimiento, en el marco del diálogo de saberes, pensamiento universal y producción científica tecnológica global (art. 351).

\section{Sintesis comparativa}

La educación superior, tanto en Uruguay como en el Ecuador, es considerada como un bien público y un derecho de las personas. En este sentido, ambos países mantienen una visión similar, sin embargo, existen algunas diferencias en cuanto a la relevancia concedida según la fuente normativa. Mientras que en Uruguay la CRU solamente menciona la declaración de utilidad pública de la gratuidad de la enseñanza superior y la garantía de la libertad de enseñanza, la CRE concibe a la educación en general como un derecho y un deber ineludible e inexcusable del Estado. Esa declaración en el caso uruguayo ocurre en la LGE, como parte de la educación en general.

La incorporación como un derecho de carácter constitucional en el Ecuador hace que pueda ser tutelado a través de la justicia constitucional. En el caso de Uruguay, a pesar de no estar expresamente determinado a ese nivel, la CRU establece que "la enumeración de derechos, deberes y garantías hecha por la Constitución, no excluye los otros que son inherentes a la personalidad humana o se deriven de la forma republicana de gobierno" (art. 72). En tal sentido, al ser la educación un derecho reconocido en el PIDESC ratificado por Uruguay, sí sería posible su tutela a través de la acción de amparo establecida en la Ley $\mathrm{N}^{\circ} 16011$ del 19 de diciembre de 1988. Por lo que, en definitiva, la educación superior mantiene el mismo estatus de derecho y bien público en ambos ordenamientos jurídicos.

La consideración de bien público trae consigo, entre otros, el reto de la democratización en su acceso y una mayor atención desde la política pública para evitar una orientación hacia los intereses privados y mercantiles. Sin embargo, las estrategias adoptadas en ambos países guardan diferencias, por ejemplo, la consagración de la autonomía en Uruguay es bastante amplia, mostrando resultados positivos en su entorno, mientras que en el Ecuador a 
la autonomía se la ha caracterizado como "responsable", desde la perspectiva de una nueva ética de las IES (Instituciones de Educación Superior) del Ecuador para actuar con miras a la vinculación y diálogo con la sociedad, y que implica una mayor presencia estatal a través de órganos de control y coordinación de las IES.

\section{Acceso a la educación superior en el ordenamiento jurídico de Uruguay y Ecuador}

Tanto Uruguay como Ecuador han suscrito y ratificado el PIDESC (1966), que al formar parte de los derechos internos de ambos países, implica el reconocimiento dado por estos al "derecho a toda persona a la educación" (art.13, $\left.\mathrm{n}^{\mathrm{o}} 1\right)$. Para alcanzar el pleno ejercicio de ese derecho "la enseñanza superior debe hacerse igualmente accesible a todos, sobre la base de la capacidad de cada uno, por cuantos medios sean apropiados, y en particular por la implantación progresiva de la enseñanza gratuita" (art. 13, $\mathrm{n}^{\circ} 2$-c).

El Comité de Derechos Económicos, Sociales y Culturales (CDESC) determina que para que el derecho a la educación superior se ejerza plenamente, deben existir los elementos de disponibilidad, accesibilidad, aceptabilidad y adaptabilidad. El elemento de disponibilidad se enfoca en la existencia de IES y programas en funcionamiento para responder a la demanda en base a las capacidades de los ciudadanos. El elemento de accesibilidad implica la garantía de no discriminación para el ingreso a la educación superior, asegurando las facilidades físicas y ubicación geográfica, y la eliminación de barreras económicas. El elemento de aceptabilidad tiene que ver con el cumplimiento de estándares mínimos de calidad. Finalmente, el elemento de adaptabilidad está relacionado con la flexibilidad de acoplarse, en el tiempo, a las necesidades y cambios en las sociedades (CDESC, 2013, p. 9, 10, 14).

En tal contexto el siguiente análisis se realizará considerando la accesibilidad a partir de la gratuidad y los sistemas de ingreso como eliminación de las barreras económicas.

\section{La gratuidad como eliminación de barreras económicas}

Uruguay y la gratuidad absoluta. El acceso a la educación superior se caracteriza por ser gratuito y al respecto la CRU señala: 
Declárase de utilidad social la gratuidad de la enseñanza oficial primaria, media, superior, industrial y artística y de la educación física, la creación de becas de perfeccionamiento y especialización cultural, científica y obrera, y el establecimiento de bibliotecas populares. En todas las instituciones docentes se atenderá especialmente la formación del carácter moral y cívico de los alumnos (art. 71).

Al respecto, Justino Jiménez Aréchaga (en Biasco, 2001, p. 13) señala que tal norma es una mera proclamación de aspiraciones, puesto que la gratuidad de la enseñanza en el Uruguay ha existido desde siempre. El autor mencionado considera a la declaratoria de utilidad social una norma de carácter programático, en el sentido de recomendación para que el legislador la considere al momento de expedir las leyes. De forma que la LGE señala que la educación pública estatal se rige por el principio de gratuidad (art. 15).

La Ley de la Universidad de la República es más expresa en su redacción, al afirmar que la enseñanza oficial es gratuita y los estudiantes no deben pagar ningún tipo de derecho o matrícula (art. 66).

La condición de norma programática mas no prescriptiva del artículo 71 de la CRU, se ratifica al observar la excepción establecida en la Ley $\mathrm{N}^{\circ}$ 16.226 del 29 octubre de 1991 (sobre rendición de cuentas y balance de ejecución presupuestal, correspondiente al ejercicio 1990) en donde se faculta a la Universidad de la República a cobrar una matrícula a los estudiantes que tengan las condiciones económicas para pagarla (art. 407).

Ecuador y la gratuidad con responsabilidad académica. La CRE establece que la educación superior pública será gratuita hasta el tercer nivel y que se vinculará a la responsabilidad académica de los estudiantes (art. 356). Esa norma contiene una prescripción condicionada. Por un lado, al disponer la gratuidad existe una prohibición de cobro a los estudiantes en las instituciones de educación superior públicas, pero en el marco de la responsabilidad académica. Es en este punto que la CRE da margen al legislador para que desarrolle el contenido de dicho criterio y así lo hace la LOES, que fija los parámetros a observarse para gozar de la gratuidad. Entre los más relevantes están: a) estudiantes que se matriculen en al menos el 60\% de las materias en cada periodo académico, b) solo se cubre las primeras matrículas ordinarias, c) se financia una sola carrera, d) solo se cubre los rubros obligatorios e indispensables para cursar y obtener la titulación, y e) se pierde la gratuidad de manera definitiva si el estudiante reprueba el 30\% de las materias cursadas (art. 80). 


\section{Sintesis comparativa}

Ambos ordenamientos establecen el acceso gratuito a la educación superior en el sistema público, una característica relevante, tomando en cuenta que la gratuidad es considerada por el PIDESC como una forma de alcanzar el pleno ejercicio del derecho a la educación. No obstante, existen algunas diferencias. La gratuidad de la educación superior pública en Uruguay no se encuentra prescrita a nivel constitucional, pero ha sido una realidad que acompaña a la universidad uruguaya desde su nacimiento, por lo que, aunque puede considerarse jurídicamente más frágil, el consenso social sobre el tema parece garantizar su permanencia. En contraste, en el Ecuador la gratuidad existe hace apenas diez años, tanto a nivel normativo-constitucional como en los hechos. En términos jurídicos, tiene mayor fortaleza que en el caso uruguayo y constituye una reivindicación lograda por los movimientos estudiantiles en un escenario político favorable para una agenda pro derechos.

Por otra parte, mientras la gratuidad en Uruguay es absoluta, en Ecuador está condicionada al criterio de responsabilidad académica. Este punto puede estar influenciado por las diferencias económicas y poblacionales de ambos Estados. Uruguay tiene una población estudiantil ${ }^{4}$ significativamente menor a la que existe en Ecuador, con un PIB per cápita significativamente mayor al de Ecuador, ${ }^{5}$ en tal sentido, le resulta posible disponer de los recursos en términos absolutos para garantizar la gratuidad de manera incondicionada. En Ecuador, a pesar de los esfuerzos para la inversión en educación superior - que incluso ha superado el porcentaje promedio de los países de la OCDE (Ballas, 2016, p. 90) - , los recursos deben ser cuidadosamente administrados. Por otro lado, se considera que si bien las personas tienen derecho a la educación pública gratuita, también es necesaria una retribución al esfuerzo social encaminado a través del Estado para su formación, y que esa retribución se traduzca principalmente en el esfuerzo y cumplimiento de las responsabilidades académicas. Es decir, el aspecto de la responsabilidad académica implica una nueva ética social que busca la superación de la mirada individual liberal a través de la integración de esfuerzos para alcanzar objetivos colectivos.

4 La matrícula de educación superior en Uruguay a 2014 fue de 165 mil personas (Presidencia de Uruguay, 2016).

5 PIB per cápita: Ecuador USD 5 968,98 (BM, 2016), Uruguay USD 15 220,57 (BM, 2016). 


\section{Sistema de ingreso en el ordenamiento jurídico}

Uruguay y el libre ingreso sin discriminación. En Uruguay generalmente la educación superior pública tiene libre ingreso, esto es, la inexistencia de un examen nacional o institucional o cualquier otro mecanismo de selección para ser aceptado en una institución pública. No se ha encontrado ninguna norma que lo prescriba de esa manera, sin embargo, en la noción de máxima autonomía, la Universidad de la República no aplica exámenes para el ingreso, en consecuencia, todos los aspirantes que deseen estudiar pueden hacerlo; así lo manifiesta Martínez (2003, p. 38), señalando, además, que otras instituciones de educación terciaria pública sí realizan algún proceso de selección por falta de cupos.

Ecuador y el sistema de nivelación y admisión para garantizar meritocracia y eficiencia. En el Ecuador el ingreso a la educación superior pública no es libre. La LOES determina un sistema unificado de acceso a la educación superior a través de un examen nacional y un periodo de nivelación de conocimientos debido a la heterogeneidad de los resultados de aprendizaje de la educación media. Este sistema es administrado por la Secretaría de Educación Superior, Ciencia, Tecnología e Innovación (SENESCYT). Los cupos son asignados en base a: el puntaje obtenido, la selección por parte del futuro estudiante de un número de posibles opciones de IES y el número de plazas disponibles dentro de esa selección (art. 81). ${ }^{6}$

Las IES privadas que reciben recursos del Estado deben poner a disposición de este sistema de acceso un número determinado de plazas (art. 74) y respecto a los demás cupos, al igual que el resto de las IES privadas, pueden aplicar sus propias reglas de ingreso, siempre que estas no sean de carácter discriminatorio conforme lo determina el RGLOES (art. 4), además de conceder un número de becas equivalente al $10 \%$ del total de su matrícula para personas con alto rendimiento académico, deportivo o pertenecientes a grupos históricamente excluidos (art. 77).

\section{Sintesis comparativa}

En lo que respecta al sistema de ingreso, a nivel de ordenamiento jurídico no es posible efectuar una comparación puesto que Uruguay no cuenta

6 Actualmente, en la Asamblea Nacional se encuentra en tratamiento un proyecto de reforma a la Ley Orgánica de Educación Superior del Ecuador, que podría modificar el sistema de ingreso a las IES en este país. 
con reglas relacionadas al tema. Podría señalarse que la ausencia de un sistema de ingreso implica una mayor democratización en el acceso, puesto que elimina cualquier discriminación del origen y la educación previa de los aspirantes a la educación superior; esto sumado a la gratuidad, se traduce en la ausencia de barreras para acceder a la formación superior. En contraste con esta idea, Germán Rama señala que:

El hecho de que la universidad no tenga un proceso de selección no quiere decir que este no exista. Existe y es el más duro, es el fracaso. Es también el más caro desde el punto de vista humano y material (en Martínez, 2003, p. 38).

No obstante, desde el punto de vista del derecho a la educación superior, en relación a las condiciones para el acceso — gratuidad y libre ingreso-, el esquema uruguayo garantiza un alto grado de igualdad de oportunidades para el acceso. Esto no quiere decir que por ello no sea necesario considerar otros aspectos que no son materia de este trabajo, como la homogeneidad de los resultados de aprendizaje de la educación media y los costos de oportunidad entre los estudios y el trabajo para los jóvenes de los estratos más pobres de la sociedad.

Por otro lado, el sistema de Ecuador intenta alcanzar un equilibrio entre la eficiencia económica, la igualdad de oportunidades, la meritocracia y la calidad. En los esfuerzos por construir una sociedad más justa e igualitaria, la educación superior tiene un lugar privilegiado, pero no es el único ámbito que requiere atención del Estado. Así, los recursos públicos para este sector se han duplicado en diez años (Ballas, 2016, p. 92), pero lograr una armonía entre la demanda y oferta de cupos en las IES es una tarea compleja, pues la primera supera a la segunda. En tales condiciones, el libre ingreso podría implicar el detrimento de la calidad (Ramírez, 2017, p. 25). Frente a recursos escasos, a aumentar la capacidad física del sistema y a incrementar la calidad, resulta necesario un mecanismo que procure equilibrio, viabilidad y la mayor justicia posible. Así, el examen busca identificar a los ciudadanos con mayores aptitudes para la formación superior y la nivelación pretende igualar los conocimientos de partida que pueden tener diferencias por la heterogeneidad de la educación media (Araujo, 2016, pp. 139-147). No obstante, existen posiciones contrarias que cuestionan el examen, entre otros aspectos, por considerar que es un mecanismo discriminador puesto que no se evalúa solo aptitudes, sino también conocimientos, por tanto, aquellos 
que no tuvieron la oportunidad de aprehender esos conocimientos estarían siendo discriminados (Zambrano, 2016, p. 11).

En todo caso, a pesar de que este sistema no tiene el mismo grado de democratización de Uruguay, significa un avance autorreferencial en la materia, considerando que existe desde el año 2010; previo a eso, cada IES definía sus propios procesos de selección bajo diferentes perspectivas y objetivos que dieron como resultado una discriminación negativa por origen socioeconómica. De tal suerte, Ramírez (2016) señala que "la posibilidad de entrar a la educación superior siendo parte del 20\% más pobre en el 2014 es del 67\% en tanto que en el 2006 era del 33\%" (p. 20). En este sentido, desde una perspectiva del derecho a la educación superior en el Ecuador, este sistema representa un importante avance en la eliminación de barreras económicas y por tanto en mejorar las condiciones para el pleno ejercicio de ese derecho.

\section{Conclusiones}

\section{Educación como bien público y derecho de las personas}

Si bien los ordenamientos jurídicos uruguayo y ecuatoriano se asemejan al considerar a la educación superior como un bien público y un derecho de las personas, se diferencian en la jerarquía de la norma jurídica en la que se efectúa este reconocimiento. Mientras en el Ecuador el carácter de bien público y derecho se encuentra a nivel constitucional, en Uruguay halla su basamento en la LGE como una declaración aplicable a todos los niveles de educación; no obstante, esta distinción no limita la posibilidad de que el derecho sea tutelado jurisdiccionalmente, puesto que la CRU extiende su protección a los derechos inherentes a la personalidad humana.

\section{Acceso gratuito a la educación superior pública}

Los ordenamientos jurídicos uruguayo y ecuatoriano guardan similitud en cuanto al establecimiento de la gratuidad de la educación superior en el sistema público y se diferencian, una vez más, en la jerarquía normativa del instrumento que la contempla. La Constitución del Ecuador es expresa al 
consagrar la gratuidad de la educación superior, mientras que en Uruguay existe la declaración de utilidad pública de la gratuidad a nivel constitucional y a nivel legal el principio de gratuidad.

Otra diferencia relevante es que la gratuidad en Uruguay es incondicional en todos los niveles, mientras que en Ecuador depende de la responsabilidad académica del estudiante y se aplica a una sola carrera de tercer nivel.

\section{Sistema de ingreso a la educación superior}

En lo que se atiene a los sistemas de ingreso a la educación superior, pese a que Uruguay no contempla normas al respecto en su legislación, en la práctica, lo que acontece es que se evidencia un acceso es libre e incondicionado, mientras tanto en Ecuador se ha desarrollado un sistema reglado que procura eficiencia económica, igualdad de oportunidades, meritocracia y calidad.

\section{Bibliografía}

Araujo, L. (2016). El Sistema Nacional de Nivelación y Admisión en Ecuador. En R. Ramírez (ed.), Universidad urgente para una sociedad emancipada (pp. 127-162). Quito: IESALC.

BCE. Estadísticas. Recuperado de https://goo.gl/doFWco/

BM. Datos Banco Mundial. Recuperado de https://goo.gl/34gGqn/

Ballas, C. (2016). Financiamiento de la educación superior en Ecuador. En R. Ramírez (ed.), Universidad urgente para una sociedad emancipada (pp. 84-106). Quito: IESALC.

Biasco, E. (s/f). La gratuidad de la enseñanza pública superior en el Uruguay. Recuperado de https://goo.gl/8FbRYw/

CEAACES. Lista de institutos técnicos y tecnológicos. Recuperado de www.ceaaces.gob.ec

Consejo de Educación Primaria. (8 de enero de 2018). Fundamentos generales para un programa de educación común. Recuperado de https://goo.gl/tbyLaL/

CES. Universidades y Escuelas Politécnicas. Recuperado de www.ces.gob.ec

De Almeida, C. y Carvalho, J. (2017). Introdução ao Direito Comparado. Coimbra: Almedina.

CDESC. (2013). Right to Education: Scope and Implementation. UNESCO. Recuperado de https://bit.ly/2KrTRYs/ 
Contera, C. (2008). La educación superior en Uruguay. Avaliaçao, 13(2), 533-554. González, M., Howard, W., Vidal, K. y Bellin, C. (2017). Manual de derecho civil. Montevideo: Universidad de la República de Uruguay.

Guijarro, J. (2016). Autonomía universitaria: política no metafísica. En R. Ramírez (ed.), Universidad urgente para una sociedad emancipada (pp. 241262). Quito: IESALC.

INE. (s/f). Uruguay en cifras. Montevideo: INE. Recuperado de https://goo.gl/jfQVtk/ INE. (s/f). Estadísticas Uruguay. Recuperado de https://goo.gl/U47fHo/ INEC. (s/f). Resultados del Censo 2010. Recuperado de https://goo.gl/yJ1rw4/ Instituto Uruguay XXI. (2011). Concentración de las exportaciones de Uruguay y las de sus competidores. Recuperado de https://goo.gl/byDhjE/

Martínez, E. (2003). La educación superior de Uruguay en transición: inercias y horizontes de cambio. Montevideo: IESALC/UNESCO.

Minteguiaga, A. y Ramírez, R. (2010). Transformaciones en la educación superior ecuatoriana: antecedentes y perspectivas futuras como consecuencia de la nueva Constitución política. Las transformaciones de la educación superior en América: Identidades en construcción. Educación Superior y Sociedad, 1, 129-154. Recuperado de https://goo.gl/Tvr4wG/

Moura Vicente, D. (2012). Direito Comparado. Coimbra: Almedina.

OCDE. (8 de enero de 2018). Distribución del ingreso por pobreza. Recuperado de https://goo.gl/cEfnGe/

Pacheco, L. (2015). La primera evaluación de la universidad ecuatoriana (19801988). Quito: CES.

Ramírez, R. (2013). Tercera ola de transformación de la educación superior en el Ecuador: hacia la constitucionalización de la sociedad del buen vivir. Quito: SENESCYT.

Ramírez, R. (ed.). (2016). Universidad Urgente para una Sociedad emancipada. Quito: IESALC.

Ramírez, R. (2017). La gran transición: en busca de nuevos sentidos comunes. Quito: CIESPAL.

Ramírez, R. (28 de enero de 2017). Rompiendo con la ley de la gravedad económica: primero los pobres, las grandes mayorías y "ajuste a la inversa”. Recuperado de reneramirez.ec.

SIISE. Distribución del consumo: coeficiente de Gini. Recuperado de https://goo. $\mathrm{gl} / 3 \mathrm{bZWmx} /$

QS Universities. (s/f). QS World University Rankings Latin America. Recuperado de https://goo.gl/cL2CKj/ 
UNESCO. (2015). Revisión regional 2015 de la educación para todos: América Latina y el Caribe. Recuperado de https://goo.gl/hrXnv7/

UNESCO. (2018). Enrolment by level of education and type of institution. Recuperado de https://goo.gl/jvyCsw/

Zambrano, J. (2016). Una mirada crítica al examen nacional para la educación superior en Ecuador. EduSol, 16(56), 37-51.

\section{Fuentes normativas de Uruguay}

Código Civil de Uruguay No 16603 (1994).

Constitución de la República Oriental de Uruguay (1830).

Constitución de la República Oriental de Uruguay (1918).

Constitución de la República Oriental de Uruguay (1934).

Constitución de la República Oriental de Uruguay (1952).

Constitución de la República Oriental de Uruguay (1967).

Decreto de Uruguay No 308/995 (1995).

Decreto de Uruguay No 104/014 (2014).

Ley de Creación de la Universidad Tecnológica de Uruguay No 19043 (2013).

Ley General de Educación de Uruguay N 18437 (2009).

Ley de Instrucción Pública de Uruguay No 1350 (1877).

Ley de Presupuesto Nacional de Uruguay 2016-2020 No 19355 (s/f).

Ley de Rendición de Cuentas y Balance de Ejecución Presupuestal del Ejercicio $1990 \mathrm{~N}^{\mathrm{o}} 16226$ (1991).

Ley Orgánica de la Universidad de la República de Uruguay (1958).

Ley sobre disposiciones para los títulos profesionales que otorguen las universidades privadas de Uruguay $\mathrm{N}^{\circ} 15661$ (1984).

Pacto Internacional de Derechos Económicos, Sociales y Culturales (1966).

\section{Fuentes normativas del Ecuador}

Código Civil de Ecuador (2005).

Constitución Política del Ecuador (1835).

Constitución Política del Ecuador (1861).

Constitución Política del Ecuador (1897).

Constitución Política del Ecuador (1929).

Constitución Política del Ecuador (1945).

Constitución Política del Ecuador (1967).

Constitución Política del Ecuador (1979).

Constitución Política del Ecuador (1998).

Constitución Política del Ecuador (2008). 
Ley del Fondo Permanente de Desarrollo Universitario y Politécnico de Ecuador (1996).

Ley de Universidades y Escuelas Politécnicas de Ecuador (1982).

Ley Orgánica de Educación Superior de Ecuador (2000).

PIDESC Pacto Internacional de Derechos Económicos, Sociales y Culturales (1966).

Reglamento General a la Ley Orgánica de Educación Superior de Ecuador (2011).

Fecha de recepción: 2018/04/01; Fecha de aceptación: 2018/07/24;

Fecha de publicación: 2018/09/01 